\author{
Roman Sykora \\ Jiri Chvojka \\ Ales Krouzecky \\ Jaroslav Radej \\ Thomas Karvunidis \\ Veronika Varnerova \\ Ivan Novak \\ Martin Matejovic
}

\section{High versus standard-volume haemofiltration in hyperdynamic porcine peritonitis: effects beyond haemodynamics?}

Received: 16 April 2008

Accepted: 15 September 2008

Published online: 14 October 2008

(C) The Author(s) 2008. This article is published with open access at Springerlink.com

Roman Sykora and Jiri Chvojka contributed equally to this study.

Electronic supplementary material The online version of this article (doi: 10.1007/s00134-008-1318-y) contains supplementary material, which is available to authorized users.
R. Sykora · J. Chvojka · A. Krouzecky · J. Radej · T. Karvunidis · V. Varnerova . I. Novak · M. Matejovic (®) ICU, 1st Medical Department, Charles University Medical School and Teaching Hospital Plzen, Alej svobody 80,

30460 Plzen, Czech Republic e-mail: matejovic@fnplzen.cz

Tel.: +420-377103501

Fax: +420-377103506

\begin{abstract}
Objective: The role of haemofiltration as an adjunctive treatment of sepsis remains a contentious issue. To address the role of dose and to explore the biological effects of haemofiltration we compared the effects of standard and high-volume haemofiltration (HVHF) in a peritonitis-induced model of porcine septic shock. Design and setting: Randomized, controlled experimental study. Subjects:
\end{abstract}

Twenty-one anesthetized and mechanically ventilated pigs.

Interventions: After $12 \mathrm{~h}$ of hyperdynamic peritonitis, animals were randomized to receive either supportive treatment (Control, $n=7$ ) or standard haemofiltration (HF $35 \mathrm{ml} /$ $\mathrm{kg}$ per h, $n=7)$ or HVHF (100 ml/kg per hour, $n=7)$. Measurements and results: Systemic and hepatosplanchnic haemodynamics, oxygen exchange, energy metabolism (lactate/pyruvate, ketone body ratios), ileal and renal cortex microcirculation and systemic inflammation (TNF- $\alpha$, IL-6), nitrosative/oxidative stress (TBARS, nitrates, GSH/GSSG) and endothelial/coagulation dysfunction (von Willebrand factor, asymmetric dimethylarginine, platelet count) were assessed before, 12, 18, and $22 \mathrm{~h}$ of peritonitis. Although fewer haemofiltration-treated animals required noradrenaline support (86, 43 and $29 \%$ animals in the control, HF and HVHF groups, respectively), neither of haemofiltration doses reversed hyperdynamic circulation, lung dysfunction and ameliorated alterations in gut and kidney microvascular perfusion. Both HF and HVHF failed to attenuate sepsisinduced alterations in surrogate markers of cellular energetics, nitrosative/oxidative stress, endothelial injury or systemic inflammation. Conclusions: In this porcine model of septic shock early HVHF proved superior in preventing the development of septic hypotension. However, neither of haemofiltration doses was capable of reversing the progressive disturbances in microvascular, metabolic, endothelial and lung function, at least within the timeframe of the study and severity of the model.

Keywords Sepsis - Haemofiltration · High volume haemofiltration . Microcirculation · Energy metabolism - Oxidative stress 


\section{Introduction}

Extracorporeal blood purification techniques as an adjunctive treatment of sepsis in critically ill patients remain a conceptually sound but still unresolved issue [1-3]. Haemofiltration, which is nowadays one of the most widely used hemopurification method in the ICU, failed to significantly reduce plasma cytokine concentrations and ameliorate organ dysfunction in septic patients when low ultrafiltration doses $(2 \mathrm{l} / \mathrm{h}$ of plasma water exchange) were used [4]. In contrast, the importance of dialysis intensity to improve the outcome of critically ill patients with acute renal failure has been demonstrated [5-7]. Nonetheless, although the minimal ultrafiltration rate of $35 \mathrm{ml} / \mathrm{kg}$ per hour has been recommended $[1,3]$, recent large multicenter trial, in which $63 \%$ of patients were septic, challenged the issue of a dosage-outcome relationship in patients with acute kidney injury [8]. In addition, Ronco et al. [5] suggested a beneficial trend in survival when further increasing the dose of haemofiltration up to $45 \mathrm{ml} / \mathrm{kg}$ per hour in a small subgroup of septic patients. Although limited in their robustness and methodological aspects, several human [9-15] and animal [16-20] studies further support the concept of high-volume haemofiltration (HVHF) in the treatment of endotoxemia or septic shock, mainly by demonstrating a reduced need for vasopressors dose and better hemodynamic stability. However, human studies were either uncontrolled or retrospective, thereby limiting the inference that could be drawn [21]. Similarly, the clinical relevance of some experimental studies focusing predominantly on haemodynamics, gas exchange, plasma cytokine levels, immunoparalysis and short-term survival, is limited by the fact that (1) haemofiltration was started before, during or very early after the septic or endotoxemic insult [21]; (2) the treatment effects were investigated mostly in hypodynamic models characterized by hypotension and low cardiac output, which is in contrast to the hyperdynamic pattern commonly seen in patients with septic shock. Finally, all studies compared the effects of HVHF either with untreated subjects or with low volume haemofiltration (i.e. $20 \mathrm{ml} / \mathrm{kg}$ per hour); hence, it is unknown whether increasing the dose of plasma water exchange confers an additional benefit over currently accepted standard haemofiltration dose (i.e. $35 \mathrm{ml} / \mathrm{kg}$ per hour). Therefore, to further explore the biological effects of haemofiltration and the role of its dose in sepsis, we investigated (1) the impact of haemofiltration on regional and microcirculatory perfusion, energy metabolism, oxidative stress, endothelial dysfunction and systemic inflammatory response in a porcine model of hyperdynamic fecal peritonitis-induced septic shock that integrates standard day-to-day care resuscitative measures; (2) whether increasing haemofiltration dose from standard (35 ml/kg per hour) to high volume $(100 \mathrm{ml} / \mathrm{kg}$ per hour) provides an additional benefit.

\section{Material and methods}

The experiment was performed in adherence to the National Institutes of Health Guidelines on the Use of Laboratory Animals. The study protocol was approved by the University Animal Care Committee. Twenty-one anesthetized and ventilated pigs of either gender with a body weight [median (quartiles): $38 \mathrm{~kg}(34,42)$ ] were investigated. The anesthesic procedure, surgical instrumentation, and physiological measurements were performed as recently described (see the Electronic supplement material for details) [22-24]. Hemodynamic parameters recorded over a period of $22 \mathrm{~h}$ comprised mean arterial pressure, cardiac output, intrathoracic blood volume (ITBV), filling pressures, portal venous, hepatic and renal arterial blood flows. Microvascular measurements included ileal mucosal and renal cortex microvascular perfusion [laser Doppler flowmetry and the Side-stream Dark Field (SDF) imaging] [22-26]. Arterial, portal, hepatic and renal venous lactate $(\mathrm{L})$ and pyruvate $(\mathrm{P})$ concentrations, arterial serum levels of alanine aminotransferase (ALT), the hepatic venous ketone body ratio (KBR, acetoacetate/ $\beta$-hydroxybutyrate), arterial thiobarbituric acid reactive species (TBARS), reduced and oxidized glutathione (GSH/GSSG), and nitrate + nitrite $(\mathrm{NO} x)$ concentrations were measured. From arterial blood samples we also assessed von Willebrand factor (vWF), platelet count, asymmetric dimethylarginine (ADMA), tumor necrosis factor alpha (TNF- $\alpha$ ), and interleukin 6 (IL-6). To correct for dilutional effects resulting from volume resuscitation NOx, TBARS, ADMA, ALT, vWF, IL- 6 and TNF- $\alpha$ levels were normalized for plasma protein content [22-24]. Finally, ultrafiltrate TNF- $\alpha$ and IL-6 concentrations were analyzed.

\section{Haemofiltration setting}

The zero-balanced haemofiltration was performed on the MultiFiltrate system (Fresenius Medical Care AG \& Co. KGaA, Bad Homburg, Germany) with a synthetic membrane (Ultraflux AV600S; the high-flux polysulphon filter, surface $1.4 \mathrm{~m}^{2}$, cut off up to 30,000 Da, Fresenius). Blood flow rate was set at $200 \mathrm{ml} / \mathrm{min}$. Bicarbonate buffered $(35 \mathrm{mmol} / \mathrm{l})$ replacement fluid (Medisol Bi-4, solution for haemofiltration, Medites Pharma, Roznov pod Radhostem, Czech Republic) was administered 50\% in predilution and $50 \%$ in postdilution fashion. The replacement fluid was warmed to $39^{\circ} \mathrm{C}$ in the MultiFiltrate, and the HOTLINE ${ }^{\circledR}$ Blood and Fluid Warmer 
(Smiths Medical, UK) was inserted on the venous return line to prevent an extracorporeal circuit-induced decrease in body temperature. Anticoagulation was achieved with an initial i.v. bolus $0.2 \mathrm{ml} / 10 \mathrm{~kg}$ of nadroparinum (Fraxiparine, Glaxo Group Ltd, Greenford, Middlesex, UK) for all experimental animals, including the control group.

\section{Protocol}

After baseline data measurements, peritonitis was induced by inoculating $0.5 \mathrm{~g} / \mathrm{kg}$ of autologous feces suspended in $200 \mathrm{ml}$ saline into the abdominal cavity through the drainage tubes. A second set of measurements was obtained after $12 \mathrm{~h}$ of peritonitis induction. Then, the pigs were randomly divided into three groups: control animals (CON $n=7$ ), animals receiving continuous haemofiltration at standard ultrafiltration rate of $35 \mathrm{ml} / \mathrm{kg}$ per hour (HF $n=7$ ) and animals treated by HVHF at an ultrafiltration rate of $100 \mathrm{ml} / \mathrm{kg}$ per hour (HVHF $n=7)$. Further measurements were recorded at 18 and $22 \mathrm{~h}$ of peritonitis. In addition to the Plasma Lyte solution, 6\% hydroxyethyl starch $130 \mathrm{kDa} / 0.4$ (Voluven 6\%, Fresenius Kabi Deutschland $\mathrm{GmbH}$, Bad Homburg, Germany) was infused at a rate of $10 \mathrm{ml} / \mathrm{kg}$ per hour; $(7 \mathrm{ml} / \mathrm{kg}$ per hour if CVP or PAOP $\geq 18 \mathrm{mmHg}$ ) to maintain cardiac filling pressures $\geq 12 \mathrm{mmHg}$. Continuous i.v. noradrenaline was administered if mean arterial pressure (MAP) fell below $70 \mathrm{mmHg}$. The dose of noradrenaline was adjusted to maintain MAP 70-75 mmHg. Additional five shamoperated animals were subjected to the haemofiltration. When the last set of data had been obtained, the animals were euthanized by $\mathrm{KCl}$ injection under deep anesthesia. The protocol of the study is illustrated in electronic supplemental material (Fig. 1 ESM).

\section{Statistical analysis}

All values shown are median and interquartile range. After the exclusion of normality using the KolmogorovSmirnov test, differences within each group between values obtained before and after the induction of peritonitis were tested using the Friedman ANOVA on ranks and, subsequently, Dunn's test for multiple comparisons with the Bonferroni's correction. Differences between the groups were analyzed using the Kruskal-Wallis one-way analysis of variance on ranks.

\section{Results}

Systemic haemodynamics, oxygen exchange, acidbase data and metabolism

The cumulative amount of colloid infusion to maintain a comparable cardiac preload was not statistically different in the three groups [control group $167(154 ; 186)$, HF group $194(160 ; 210)$, HVHF $194(159 ; 202) \mathrm{ml} / \mathrm{kg}]$. Fluid resuscitation maintained ITBV and significantly increased filling pressures (data not shown) without intergroup differences. All pigs developed hyperdynamic circulation with high cardiac output and low systemic vascular resistance, not reversed by the treatment. Although not statistically significant, six animals $(86 \%)$ in the control group received noradrenaline infusion, while three $(43 \%)$ and two (29\%) pigs required vasopressor support in the HF and HVHF groups, respectively (Fig. 1). In animals requiring vasopressor support, the median time to development of arterial hypotension was not different in all groups (control group 19 [18, 20], HF group 17 [17, 20], HVHF group $18 \mathrm{~h}[17,19]$ ) (Fig. 1). The increased cardiac output resulted in a significant rise of systemic oxygen delivery without intergroup differences, while systemic oxygen consumption did not change in any of the three groups over time. The decline in arterial $\mathrm{pH}$ and $\mathrm{BE}$ was less pronounced in both haemofiltration groups, although the intergroup difference did not reach statistical difference. A significant progressive deterioration of the $\mathrm{PaO}_{2} /$ $\mathrm{FiO}_{2}$ ratio was observed in all groups, without intergroup differences. The arterial L/P ratio increased progressively in all three groups, again without intergroup differences. There were no significant differences in body core temperature between the groups over time $(P=0.079$ at $22 \mathrm{~h})$ (Table 1).

Regional haemodynamics, microcirculation, metabolism and organ function

While liver blood flow and liver oxygen delivery increased significantly in all three groups, liver oxygen uptake remained unchanged. Renal artery blood flow, oxygen delivery and consumption did not change over time in any of the experimental groups. Despite well-maintained
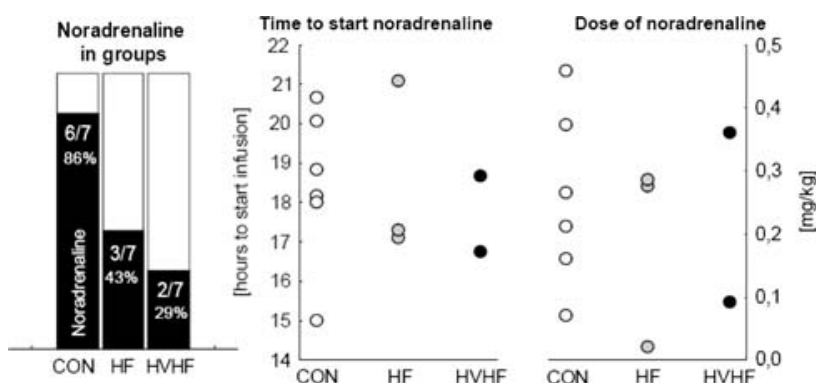

Fig. 1 Noradrenaline support. Left proportion of animals treated with noradrenaline in each group (black part of slopes); middle time to start of noradrenaline infusion after the induction of peritonitis; right total dose of administered noradrenaline; white plots designate control group $(C O N)$, grey plots designate standard haemofiltration group $(H F)$ and black plots designate high-volume haemofiltration group $(H V H F)$ 
Table 1 Systemic haemodynamics, oxygen exchange, metabolism and temperature

\begin{tabular}{|c|c|c|c|c|}
\hline & Baseline & $12 \mathrm{~h}$ & $18 \mathrm{~h}$ & $22 \mathrm{~h}$ \\
\hline \multicolumn{5}{|c|}{ MAP $(\mathrm{mmHg})$} \\
\hline $\mathrm{CON}$ & $94(89 ; 104)$ & $93(85 ; 102)$ & $85(80 ; 102)$ & $76(74 ; 92)^{\mathrm{a}}$ \\
\hline $\mathrm{HF}$ & $84(82 ; 96)$ & $88(84 ; 94)$ & $92(88 ; 103)$ & $78(71 ; 81)$ \\
\hline HVHF & $94(87 ; 100)$ & $98(84 ; 113)$ & $91(76 ; 98)$ & $81(72 ; 91)$ \\
\hline \multicolumn{5}{|c|}{$\mathrm{CO}(\mathrm{ml} / \mathrm{kg})$} \\
\hline $\mathrm{CON}$ & $70(61 ; 88)$ & $108(94 ; 122)^{\mathrm{a}}$ & $114(107 ; 128)^{\mathrm{a}}$ & $147(99 ; 183)^{\mathrm{a}}$ \\
\hline $\mathrm{HF}$ & $73(72 ; 85)$ & $125(102 ; 131)^{\mathrm{a}}$ & $129(112 ; 134)^{\mathrm{a}}$ & $136(126.162)^{\mathrm{a}}$ \\
\hline HVHF & $74(68 ; 80)$ & $106(92 ; 117)^{\mathrm{a}}$ & $111(107 ; 135)^{\mathrm{a}}$ & $131(126.155)^{\mathrm{a}}$ \\
\hline \multicolumn{5}{|c|}{ MPAP $(\mathrm{mmHg})$} \\
\hline $\mathrm{CON}$ & $26(23 ; 27)$ & $34(30 ; 36)^{\mathrm{a}}$ & $35(34 ; 40)^{\mathrm{a}}$ & $38(36 ; 43)^{\mathrm{a}}$ \\
\hline $\mathrm{HF}$ & $27(25 ; 47)$ & $30(29 ; 48)^{\mathrm{a}}$ & $34(31 ; 67)^{\mathrm{a}}$ & $34(32 ; 59)^{\mathrm{a}}$ \\
\hline HVHF & $25(22 ; 29)$ & $29(27 ; 39)^{\mathrm{a}}$ & $34(31 ; 37)^{\mathrm{a}}$ & $40(31 ; 47)^{\mathrm{a}}$ \\
\hline \multicolumn{5}{|c|}{ SVR (dyne $\mathrm{s} / \mathrm{cm}^{-5}$ ) } \\
\hline $\mathrm{CON}$ & $2774(2524 ; 2839)$ & $1717(1467 ; 1956)^{\mathrm{a}}$ & $1361(1068 ; 1837)^{\mathrm{a}}$ & $851(719 ; 1514)^{\mathrm{a}}$ \\
\hline $\mathrm{HF}$ & $2201(1914 ; 2889)$ & $1275(1191 ; 1964)^{\mathrm{a}}$ & $1276(1172 ; 1669)^{\mathrm{a}}$ & $929(858 ; 1276)^{\mathrm{a}}$ \\
\hline HVHF & $2447(2070 ; 2734)$ & $1782(1589 ; 2014)$ & $1340(1158 ; 1583)^{\mathrm{a}}$ & $1161(830 ; 1326)^{\mathrm{a}}$ \\
\hline \multicolumn{5}{|c|}{ ITBV (ml/kg) } \\
\hline $\mathrm{CON}$ & $22(19 ; 33)$ & $25(19 ; 31)$ & $26(21 ; 28)$ & $22(18 ; 30)$ \\
\hline $\mathrm{HF}$ & $25(20 ; 29)$ & $30(24 ; 30)$ & $25(21 ; 25)$ & $26(25 ; 27)$ \\
\hline HVHF & $19(17 ; 25)$ & $24(22 ; 26)$ & $26(21 ; 28)$ & $27(25 ; 28)$ \\
\hline \multicolumn{5}{|c|}{$\mathrm{DO}_{2}(\mathrm{ml} / \mathrm{min}$ per $\mathrm{kg})$} \\
\hline CON & $9(8 ; 13)$ & $15(14 ; 18)^{\mathrm{a}}$ & $16(15 ; 20)^{\mathrm{a}}$ & $24(16 ; 24)^{\mathrm{a}}$ \\
\hline $\mathrm{HF}$ & $10(8 ; 11)$ & $18(12 ; 18)^{\mathrm{a}}$ & $17(16 ; 18)^{\mathrm{a}}$ & $16(15 ; 17)^{\mathrm{a}}$ \\
\hline HVHF & $10(9 ; 11)$ & $15(13 ; 15)^{\mathrm{a}}$ & $14(13 ; 15)^{\mathrm{a}}$ & $15(13 ; 16)^{\mathrm{a}}$ \\
\hline \multicolumn{5}{|c|}{$\mathrm{VO}_{2}(\mathrm{ml} / \mathrm{min}$ per $\mathrm{kg})$} \\
\hline $\mathrm{CON}$ & $5(4 ; 6)$ & $6(5 ; 9)$ & $6(5 ; 7)$ & $7(6 ; 9)$ \\
\hline $\mathrm{HF}$ & $5(4 ; 6)$ & $6(4 ; 7)$ & $6(5 ; 7)$ & $5(5 ; 6)$ \\
\hline HVHF & $4(4 ; 5)$ & $6(5 ; 6)$ & $5(5 ; 6)$ & $5(5 ; 6)$ \\
\hline \multicolumn{5}{|c|}{ Arterial pH } \\
\hline CON & $7.52(7.50 ; 7.54)$ & $7.47(7.44 ; 7.49)^{\mathrm{a}}$ & $7.45(7.43 ; 7.50)^{\mathrm{a}}$ & $7.36(7.32 ; 7.41)^{\mathrm{a}}$ \\
\hline $\mathrm{HF}$ & $7.50(7.48 ; 7.54)$ & $7.49(7.46 ; 7.50)$ & $7.48(7.46 ; 7.49)$ & $7.45(7.40 ; 7.48)$ \\
\hline HVHF & $7.53(7.50 ; 7.55)$ & $7.46(7.43 ; 7.52)^{\mathrm{a}}$ & $7.45(7.43 ; 7.47)^{\mathrm{a}}$ & $7.40(7.36 ; 7.48)^{\mathrm{a}}$ \\
\hline \multicolumn{5}{|c|}{ Base excess $(\mathrm{mmol} / \mathrm{l})$} \\
\hline $\mathrm{CON}$ & $5.7(4.5 ; 6.6)$ & $5.5(4.7 ; 5.9)$ & $4.2(2.5 ; 5.9)$ & $1.4(-1.5 ; 1.4)^{\mathrm{a}}$ \\
\hline $\mathrm{HF}$ & $6.8(5.2 ; 8.6)$ & $7.2(5.1 ; 8)$ & $5.3(3.4 ; 6.6)$ & $4.4(0.8 ; 5.8)^{\mathrm{a}}$ \\
\hline HVHF & $7.9(5.5 ; 8.4)$ & $5.5(3.9 ; 6.2)$ & $4.3(3.2 ; 4.9)$ & $2(1.3 ; 6.4)^{\mathrm{a}}$ \\
\hline \multicolumn{5}{|c|}{ Core temperature $\left({ }^{\circ} \mathrm{C}\right)$} \\
\hline $\mathrm{CON}$ & $38.0(37.0 ; 38.3)$ & $40.2(39.1 ; 41.3)^{\mathrm{a}}$ & $39.6(38.7 ; 40.3)$ & $39.5(38.0 ; 40.4)$ \\
\hline $\mathrm{HF}$ & $38.2(37.2 ; 39.3)$ & $39.7(39.0 ; 40.5)^{\mathrm{a}}$ & $38.7(38.0 ; 38.8)$ & $38.5(38.0 ; 38.8)$ \\
\hline HVHF & $37.6(35.3 ; 38.0)$ & $39.6(37.8 ; 39.8)^{\mathrm{a}}$ & $37.7(37.4 ; 38.1)$ & $37.5(37.4 ; 38.1)$ \\
\hline \multicolumn{5}{|c|}{ Oxygenation index $\left(\mathrm{PaO}_{2} / \mathrm{FiO}_{2}\right)$} \\
\hline CON & $416(345 ; 475)$ & $409(289 ; 490)$ & $283(267 ; 396)^{\mathrm{a}}$ & $160(94 ; 271)^{\mathrm{a}}$ \\
\hline $\mathrm{HF}$ & $491(439 ; 509)$ & $365(292 ; 445)^{\mathrm{a}}$ & $349(259 ; 454)^{\mathrm{a}}$ & $238(174 ; 294)^{\mathrm{a}}$ \\
\hline HVFH & $467(396 ; 495)$ & $437(360 ; 499)$ & $289(230 ; 347)^{\mathrm{a}}$ & $156(72 ; 321)^{\mathrm{a}}$ \\
\hline \multicolumn{5}{|c|}{ Arterial lactate/pyruvate ratio } \\
\hline CON & $11(7 ; 19)$ & $18(11 ; 22)$ & $17(15 ; 36)$ & $31(20 ; 46)^{\mathrm{a}}$ \\
\hline $\mathrm{HF}$ & $12(11 ; 14)$ & $10(7 ; 14)$ & $9(8 ; 20)$ & $28(19 ; 56)^{\mathrm{a}}$ \\
\hline HVFH & $13(11 ; 17)$ & $15(8 ; 29)$ & $22(14 ; 53)^{\mathrm{a}}$ & $60(29 ; 87)^{\mathrm{a}}$ \\
\hline
\end{tabular}

$M A P$ mean arterial pressure, $C O$ cardiac output, $M P A P$ mean pulmonary artery pressure, $S V R$ systemic vascular resistance, ITBV intrathoracic blood volume, $\mathrm{DO}_{2}$ systemic oxygen delivery, $\mathrm{VO}_{2}$ systemic oxygen uptake, $C O N$ control group, $H F$ standard haemofiltration group, $H V H F$ high-volume haemofiltration group

regional blood flow both ileal mucosal and renal cortex microvascular perfusion, as assessed by laser Doppler flowmetry, fell progressively in the control and HVHF groups (Fig. 2). Although the decline in renal cortex perfusion did not reach statistical significance in the HF group, there were no significant intergroup differences between all groups at the end of the experiment (Fig. 2). Direct visualization of the ileal mucosal microcirculation a Significant difference within each group versus baseline $(P<0.05)$. Data are median and 25 th and 75 th quartiles

was consistent with laser Doppler flowmetry: Side-stream Dark Field imaging showed well perfused villi before the induction of peritonitis, while the mean flow index deteriorated over time in all experimental groups without intergroup differences. Whereas the decrease in renal and hepatic venous $\mathrm{pH}$ was less pronounced in treated animals, neither of the haemofiltration doses significantly prevented sepsis-induced changes in the hepato-splanchnic L/P ratios 

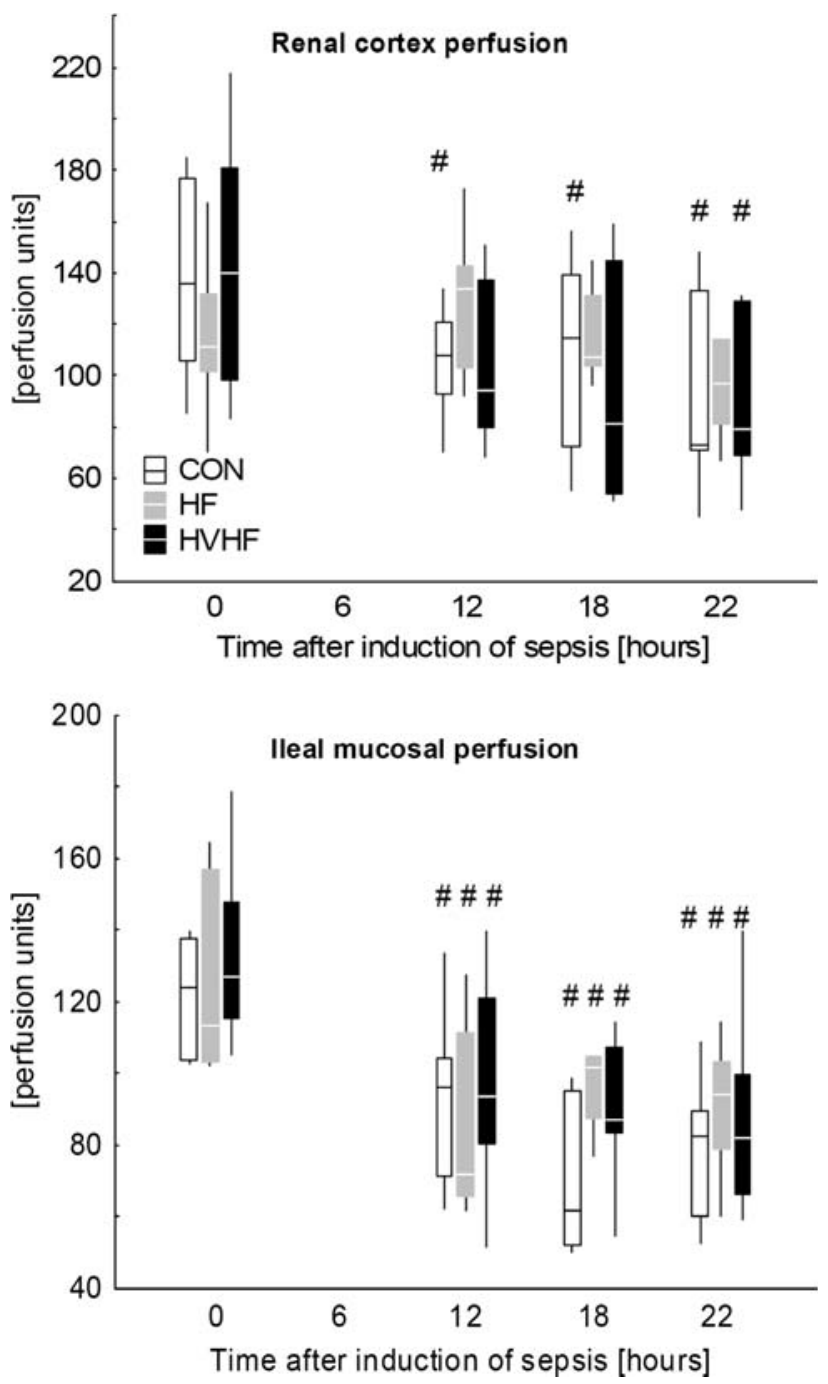

Fig. 2 Renal cortex and ileal mucosal microcirculation in the control (white plots), standard haemofiltration (HF grey plots), and high-volume haemofiltration (HVHF black plots) groups. Upper renal cortex perfusion (laser Doppler flowmetry). Lower ileal mucosal perfusion (laser Doppler flowmetry). Data are median

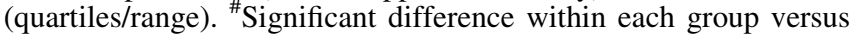
baseline $(P<0.05)$

and KBR (Fig. 3). The significant increase in serum ALT activity occurring in the control and HF animals did not reach any intergroup significance. As expected, both treatment modalities presented with lower levels in serum creatinine (Table 2).

Inflammation, oxidative/nitrosative stress and endothelial dysfunction (Table 3, Fig. 2 ESM)

Peritonitis-induced septic shock resulted in gradually increased plasma levels of TNF- $\alpha$ and IL-6 (Table 3) and overproduction of nitric oxide, as documented by a significant increase in arterial NOx levels (Fig. 2 ESM).
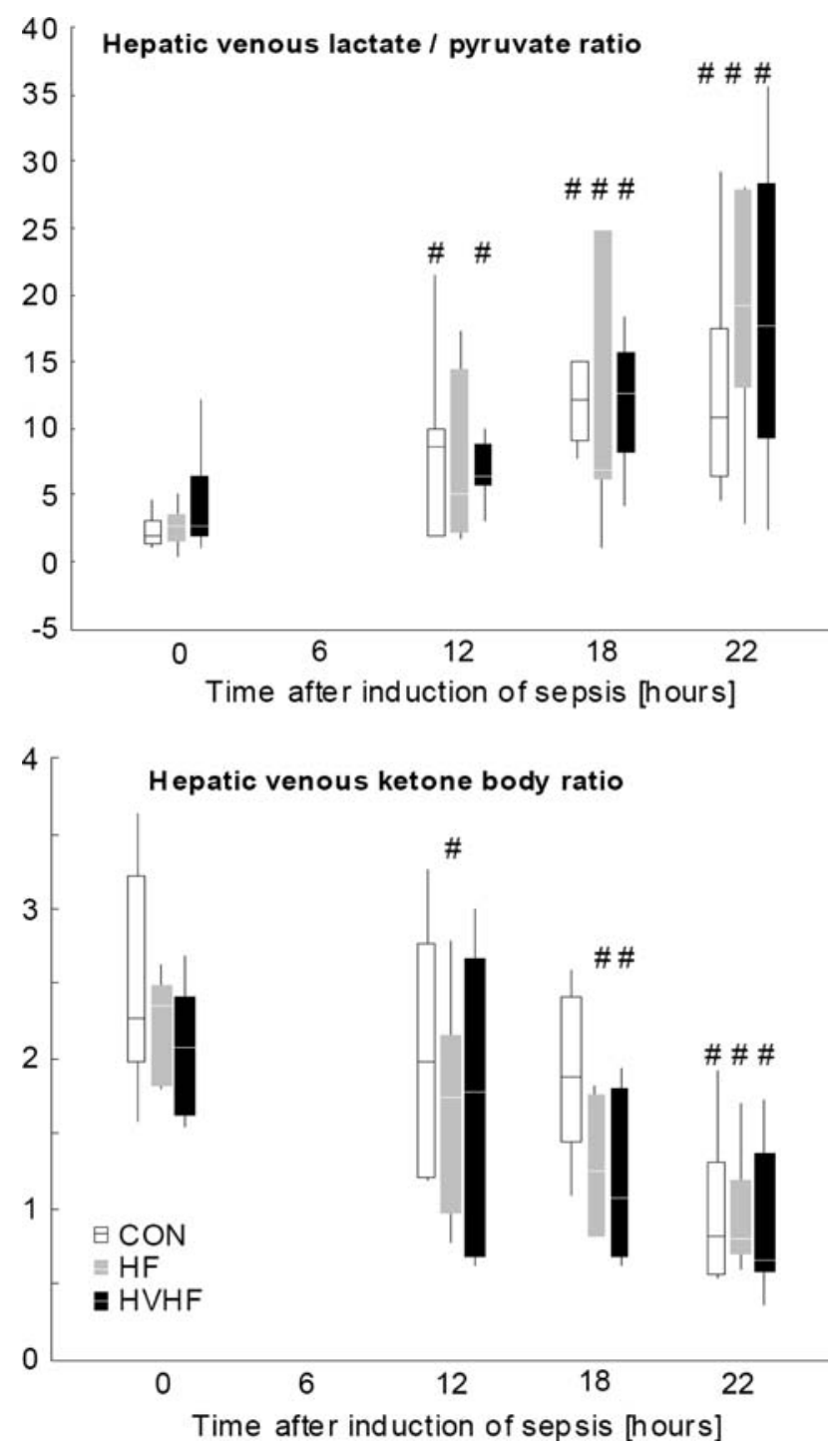

Fig. 3 Hepatic venous lactate/pyruvate ratios (upper), hepatic venous ketone body ratios (lower) in the control (white plots), standard haemofiltration (HF grey plots) and high-volume haemofiltration (HVHF black plots) groups. Data are median (quartiles/ range). ${ }^{\#}$ Significant differences within each group versus baseline $(P<0.05)$

These changes were accompanied by a several-fold increase in TBARS levels and GSH/GSSG ratio (Table 3) providing evidence for oxidative stress and an altered redox status. Conversely, ADMA concentrations decreased during the course of peritonitis (Table 3). Oxidative/nitrosative stress was paralleled by a marked increase in $\mathrm{vWf}$ suggesting altered endothelial function (Fig. 2 ESM). Both HF and HVHF did not affect the plasma levels of IL-6. Although HVHF tended to blunt the sepsis-induced increase in TNF- $\alpha$, none of haemofiltration doses influenced the surrogate markers of oxidative/nitrosative stress and endothelial dysfunction. Likewise, platelet count fell progressively in response to 
Table 2 Regional haemodynamics, oxygen exchange, acid-base status and organ function

\begin{tabular}{|c|c|c|c|c|}
\hline & Baseline & $12 \mathrm{~h}$ & $18 \mathrm{~h}$ & $22 \mathrm{~h}$ \\
\hline \multicolumn{5}{|c|}{ Q liver (ml/kg per min) } \\
\hline $\mathrm{CON}$ & $27(21 ; 28)$ & $35(31 ; 37)^{\mathrm{a}}$ & $28(27 ; 33)$ & $24(19 ; 28)$ \\
\hline $\mathrm{HF}$ & $20(20 ; 23)$ & $30(26 ; 38)$ & $33(26 ; 40)$ & $29(18 ; 38)$ \\
\hline HVFH & $27(24 ; 29)$ & $34(27 ; 38)$ & $34(31 ; 42)$ & $36(26 ; 38)$ \\
\hline \multicolumn{5}{|c|}{ Liver $\mathrm{DO}_{2}(\mathrm{ml} / \mathrm{min}$ per $\mathrm{kg})$} \\
\hline $\mathrm{CON}$ & $1.8(1.6 ; 2.2)$ & $4.4(4.1 ; 4.6)^{\mathrm{a}}$ & $4.1(3.6 ; 4.7)^{\mathrm{a}}$ & $3.4(2.9 ; 4.1)^{\mathrm{a}}$ \\
\hline $\mathrm{HF}$ & $1.3(1.0 ; 1.7)$ & $4.1(2.8 ; 4.4)^{\mathrm{a}}$ & $3.9(3.0 ; 4.7)^{\mathrm{a}}$ & $3.3(2.6 ; 4.1)^{\mathrm{a}}$ \\
\hline HVFH & $1.9(1.7 ; 2.2)$ & $3.4(3.3 ; 4.4)^{\mathrm{a}}$ & $4.0(3.5 ; 4.4)^{\mathrm{a}}$ & $3.1(2.9 ; 3.8)^{\mathrm{a}}$ \\
\hline \multicolumn{5}{|c|}{ Liver $\mathrm{VO}_{2}(\mathrm{ml} / \mathrm{min}$ per $\mathrm{kg})$} \\
\hline $\mathrm{CON}$ & $1.0(0.8 ; 1.1)$ & $1.0(0.6 ; 1.5)$ & $1.1(0.8 ; 1.2)$ & $1.1(1.0 ; 1.3)$ \\
\hline $\mathrm{HF}$ & $0.8(0.5 ; 0.9)$ & $0.9(0.7 ; 1.0)$ & $0.9(0.7 ; 1.1)$ & $1.0(0.8 ; 1.3)$ \\
\hline HVFH & $0.7(0.6 ; 0.8)$ & $1.0(0.8 ; 1.2)$ & $0.8(0.6 ; 1.0)$ & $0.8(0.7 ; 1.4)$ \\
\hline \multicolumn{5}{|l|}{ pH HV } \\
\hline $\mathrm{CON}$ & $7.44(7.43 ; 7.46)$ & $7.38(7.37 ; 7.42)^{\mathrm{a}}$ & $7.39(7.37 ; 7.41)^{\mathrm{a}}$ & $7.31(7.28 ; 7.36)^{\mathrm{a}}$ \\
\hline $\mathrm{HF}$ & $7.42(7.39 ; 7.47)$ & $7.43(7.40 ; 7.43)$ & $7.41(7.34 ; 7.43)$ & $7.41(7.35 ; 7.42)$ \\
\hline HVFH & $7.45(7.42 ; 7.49)$ & $7.36(7.34 ; 7.48)$ & $7.40(7.38 ; 7.42)$ & $7.41(7.35 ; 7.41)$ \\
\hline \multicolumn{5}{|c|}{ Serum ALT levels (nkat/g protein) } \\
\hline $\mathrm{CON}$ & $12(12 ; 15)$ & $11(11 ; 16)$ & $12(12 ; 18)^{\mathrm{a}}$ & $19(12 ; 19)^{\mathrm{a}}$ \\
\hline $\mathrm{HF}$ & $12(10 ; 13)$ & $12(11 ; 15)$ & $15(12 ; 16)^{\mathrm{a}}$ & $14(12 ; 17)^{\mathrm{a}}$ \\
\hline HVFH & $15(9 ; 16)$ & $12(11 ; 16)$ & $13(11 ; 16)$ & $15(10 ; 17)$ \\
\hline \multicolumn{5}{|c|}{$\mathrm{Q}$ renal artery (ml/kg per min) } \\
\hline $\mathrm{CON}$ & $6(4 ; 6)$ & $7(5 ; 8)$ & $7(5 ; 7)$ & $4(4 ; 6)$ \\
\hline $\mathrm{HF}$ & $5(4 ; 6)$ & $7(6 ; 10)$ & $6(5 ; 8)$ & $6(4 ; 9)$ \\
\hline HVFH & $7(3 ; 9)$ & $6(3 ; 8)$ & $4(3 ; 9)$ & $4(2 ; 10)$ \\
\hline \multicolumn{5}{|c|}{ Renal $\mathrm{DO}_{2}(\mathrm{ml} / \mathrm{min}$ per kg) } \\
\hline $\mathrm{CON}$ & $0.8(0.6 ; 0.9)$ & $1.1(0.8 ; 1.2)$ & $0.8(0.7 ; 1.1)$ & $0.6(0.5 ; 1.1)$ \\
\hline $\mathrm{HF}$ & $0.7(0.5 ; 0.8)$ & $1.0(0.7 ; 1.4)$ & $0.8(0.7 ; 1.0)$ & $0.7(0.5 ; 1.1)$ \\
\hline HVFH & $0.7(0.4 ; 1.4)$ & $0.8(0.4 ; 1.1)$ & $0.5(0.4 ; 1.0)$ & $0.5(0.2 ; 1.2)$ \\
\hline \multicolumn{5}{|c|}{ Renal $\mathrm{VO}_{2}(\mathrm{ml} / \mathrm{min}$ per $\mathrm{kg})$} \\
\hline $\mathrm{CON}$ & $0.2(0.1 ; 0.2)$ & $0.2(0.1 ; 0.2)$ & $0.2(0.2 ; 0.2)$ & $0.2(0.1 ; 0.2)$ \\
\hline $\mathrm{HF}$ & $0.2(0.1 ; 0.3)$ & $0.2(0.2 ; 0.2)$ & $0.2(0.1 ; 0.2)$ & $0.2(0.2 ; 0.3)$ \\
\hline HVFH & $0.1(0.1 ; 0.2)$ & $0.2(0.1 ; 0.2)$ & $0.1(0.1 ; 0.2)$ & $0.1(0.04 ; 0.2)$ \\
\hline \multicolumn{5}{|l|}{ pH RV } \\
\hline $\mathrm{CON}$ & $7.50(7.49 ; 7.51)$ & $7.42(7.41 ; 7.47)^{\mathrm{a}}$ & $7.46(7.43 ; 7.47)^{\mathrm{a}}$ & $7.35(7.33 ; 7.39)^{\mathrm{a}}$ \\
\hline $\mathrm{HF}$ & $7.49(7.41 ; 7.51)$ & $7.45(7.44 ; 7.48)$ & $7.46(7.40 ; 7.48)$ & $7.44(7.37 ; 7.46)^{\mathrm{a}}$ \\
\hline HVFH & $7.54(7.53 ; 7.54)$ & $7.47(7.44 ; 7.51)^{\mathrm{a}}$ & $7.43(7.43 ; 7.44)^{\mathrm{a}}$ & $7.40(7.31 ; 7.44)^{\mathrm{a}}$ \\
\hline \multicolumn{5}{|c|}{ Creatinine $(\mu \mathrm{mol} / \mathrm{l})$} \\
\hline $\mathrm{CON}$ & $91(91 ; 97)$ & $95(81 ; 118)$ & $98(88 ; 129)$ & $129(94 ; 130)$ \\
\hline $\mathrm{HF}$ & $86(79 ; 98)$ & $84(75 ; 95)$ & $75(62 ; 77)^{b}$ & $72(67 ; 77)^{b}$ \\
\hline HVFH & $88(78 ; 96)$ & $85(75 ; 95)$ & $62(56 ; 82)^{b}$ & $62(51 ; 78)^{b}$ \\
\hline \multicolumn{5}{|c|}{ Ileal mucosal microcirculation (MFI) } \\
\hline $\mathrm{CON}$ & $3.0(2.9 ; 3.0)$ & $2.9(2.2 ; 3.0)^{\mathrm{a}}$ & $2.4(2,1 ; 2,7)^{\mathrm{a}}$ & $2.2(2.0 ; 2.3)^{\mathrm{a}}$ \\
\hline $\mathrm{HF}$ & $3.0(2.9 ; 3.0)$ & $2.4(1.9 ; 2.8)^{\mathrm{a}}$ & $2.8(2.5 ; 3.0)$ & $2.5(2.4 ; 2.7)^{\mathrm{a}}$ \\
\hline HVFH & $3.0(3.0 ; 3.0)$ & $3.0(2.9 ; 3.0)^{\mathrm{a}}$ & $2.3(2.0 ; 2.5)^{\mathrm{a}}$ & $2.5(2.3 ; 2.9)^{\mathrm{a}}$ \\
\hline
\end{tabular}

$Q$ liver liver blood flow, liver $\mathrm{DO}_{2}$, liver oxygen delivery, liver $\mathrm{VO}_{2}$, liver oxygen uptake, $\mathrm{pH} H V$ hepatic venous $\mathrm{pH}, A L T$ alanine aminotransferase, $Q$ renal artery renal artery blood flow, renal $\mathrm{DO}_{2}$ renal oxygen delivery, renal $\mathrm{VO}_{2}$, renal oxygen uptake, $\mathrm{pH} \mathrm{RV}$ renal venous $\mathrm{pH}$, creatinine serum creatinine levels, MFI mean flow

peritonitis in all groups, without intergroup differences (Fig. 2 ESM). In contrast to high plasma levels of both cytokines, the concentrations of IL- 6 and of TNF- $\alpha$ in hemofiltrates from both conventional and HVHFs were close to zero (data not shown).

In sham-operated animals haemofiltration did not affect hemodynamic, microcirculatory, and metabolic data nor markers of inflammation, oxidative/nitrosative stress and endothelial dysfunction (data not shown). index, $C O N$ control group, $H F$ standard haemofiltration group, $H V H F$ high-volume haemofiltration group

a Significant difference within each group vs. baseline $(P<0.05)$

b significant intergroup differences at the same time $(\mathrm{p}<0.05)$. Data are median and 25th and 75th quartiles

\section{Discussion}

This experimental study evaluated the effects of continuous conventional and HVHF in various biological systems related to multiple pathophysiologic pathways in a porcine model of septic shock. The main findings of the present study were that (1) haemofiltration prevented the progression of septic hypotension within the study period, with the effect being more pronounced in the HVHF 
Table 3 Inflammation and oxidative/nitrosative stress

\begin{tabular}{|c|c|c|c|c|}
\hline & Baseline & $12 \mathrm{~h}$ & $18 \mathrm{~h}$ & $22 \mathrm{~h}$ \\
\hline \multicolumn{5}{|c|}{ Plasma IL-6 levels (nmol/g protein) } \\
\hline $\mathrm{CON}$ & $115^{\circ}(70 ; 205)$ & $555(361 ; 650)^{\mathrm{a}}$ & $1298(504 ; 2405)^{\mathrm{a}}$ & $2329(821 ; 9805)^{\mathrm{a}}$ \\
\hline $\mathrm{HF}$ & $72(22 ; 187)$ & $292(262 ; 426)^{\mathrm{a}}$ & $1432(487 ; 2256)^{\mathrm{a}}$ & $978(786 ; 4048)^{\mathrm{a}}$ \\
\hline HVFH & $148(110 ; 265)$ & $317(173 ; 901)^{\mathrm{a}}$ & $567(312 ; 2259)^{\mathrm{a}}$ & $985(543 ; 10771)^{\mathrm{a}}$ \\
\hline \multicolumn{5}{|c|}{ Plasma TNF- $\alpha$ levels (nmol/g protein) } \\
\hline $\mathrm{CON}$ & $1(1 ; 1)$ & $5(5 ; 9)^{\mathrm{a}}$ & $11(6 ; 18)^{\mathrm{a}}$ & $18(6 ; 23)^{\mathrm{a}}$ \\
\hline $\mathrm{HF}$ & $1(0 ; 2)$ & $5(3 ; 7)^{\mathrm{a}}$ & $6(3 ; 10)^{a}$ & $13(5 ; 18)^{\mathrm{a}}$ \\
\hline HVFH & $2(0 ; 2)$ & $3(2 ; 8)^{\mathrm{a}}$ & $5(3 ; 7)^{\mathrm{a}}$ & $6(4 ; 9)^{\mathrm{a}}$ \\
\hline \multicolumn{5}{|c|}{ Plasma TBARS levels (nmol/g protein) } \\
\hline $\mathrm{CON}$ & $19(15 ; 22)$ & $47(38 ; 60)^{\mathrm{a}}$ & $77(55 ; 89)^{\mathrm{a}}$ & $66(54 ; 98)^{\mathrm{a}}$ \\
\hline $\mathrm{HF}$ & $17(16 ; 22)$ & $50(45 ; 51)^{\mathrm{a}}$ & $60(49 ; 71)^{\mathrm{a}}$ & $72(53 ; 88)^{\mathrm{a}}$ \\
\hline HVFH & $17(15 ; 19)$ & $45(35 ; 71)^{\mathrm{a}}$ & $55(43 ; 85)^{\mathrm{a}}$ & $53(42 ; 80)^{\mathrm{a}}$ \\
\hline \multicolumn{5}{|c|}{ Plasma ADMA levels (nmol/g protein) } \\
\hline $\mathrm{CON}$ & $54(46 ; 68)$ & $87(73 ; 94)$ & $118(56 ; 141)$ & $53(46 ; 120)$ \\
\hline HF & $50(42 ; 57)$ & $53(38 ; 79)$ & $41(36 ; 97)$ & $61(45 ; 128)$ \\
\hline HVFH & $48(42 ; 59)$ & $80(71 ; 93)$ & $75(46 ; 128)$ & $97(50 ; 117)$ \\
\hline \multicolumn{5}{|c|}{ GSH/GSSG } \\
\hline CON & $9(7 ; 10)$ & $15(12 ; 25)$ & $25(13 ; 33)$ & $142(30 ; 375)^{\mathrm{a}}$ \\
\hline $\mathrm{HF}$ & $14(10 ; 19)$ & $18(15 ; 47)$ & $41(23 ; 201)$ & $148(56 ; 497)^{\mathrm{a}}$ \\
\hline HVFH & $10(8 ; 12)$ & $32(13 ; 33)$ & $28(22 ; 63)$ & $106(28 ; 204)^{\mathrm{a}}$ \\
\hline
\end{tabular}

a Significant difference within each group versus baseline $(P<0.05)$

$I L-6$ interleukin-6, TNF- $\alpha$ tumor necrosis factor alpha, TBARS plasma thiobarbituric acid reactive species, ADMA asymmetric dimethylarginine, $G S H / G S S G$ reduced/oxidized glutathione ratio,

CON control group, $H F$ standard haemofiltration group, $H V H F$ high-volume haemofiltration group

a Significant difference within each group versus baseline $(P<0.05)$

group; (2) despite favorable effects on vasopressor requirements, neither of the haemofiltration doses beneficially modulated the sepsis-induced alterations in microcirculatory perfusion and energy metabolism, endothelial dysfunction and oxidative stress.

The reduced need for vasopressor support observed in both haemofiltration groups is consistent with previous experimental $[16,19,20,27]$ and human studies $[9,11,13$, 14]. The unchanged cardiac performance and comparable cardiac preload suggest an influence of haemofiltration on pathways implicated in the control of vascular tone, such as cytokine-driven iNOS-generated overproduction of nitric oxide or opening of ATP-sensitive potassium channels [28, 29]. The former pathway, however, is unlikely since we were unable to demonstrate a clear-cut removal of proinflammatory cytokines nor a suppression of excessive NO production, as documented by the absence of an effect on plasma nitrate/nitrite levels. In addition, reactive nitrogen/ oxygen species were recently reported to be capable of inducing vascular hyporeactivity and vasodilatory hypotension [30-32]. Nevertheless, as haemofiltration failed to affect surrogate markers of sepsis-induced oxidative/ nitrosative stress, it seems conceivable to speculate that the better hemodynamic stability was not related to changes in these downstream pathways. One could also argue that the vasopressor-sparing effect of haemofiltration might simply be the result of cooling [33] and/or better acid-base control $[34,35]$ rather than a mediator-based mechanism. However, we did not observe any obvious differences in body core temperature and systemic $\mathrm{pH}$ in septic animals treated with or without haemofiltration. Admittedly, the contribution of cooling effect to the reduced need for vasopressor support cannot be entirely neglected. In support of this notion, we could recently demonstrate that even a mild haemofiltration-induced decrease in core temperature, the extent of which was comparable to this study, had the potential to increase systemic vascular resistance in septic patients [33]. In contrast, Rogiers et al. [36] observed that a decrease in body temperature during haemofiltration resulted in a rapid development of hypotension and hypodynamic circulation in ovine septic shock model. It should be noted, however, that in the Rogiers study the body temperature fell below baseline with the lowest value of $36.1^{\circ} \mathrm{C}$, which is in marked contrast to our results. Alternatively, cutting the plasma peak concentrations of cytokines [37] without a measurable effect on plasma cytokines levels or elimination of other unmeasured vasodilatory or inflammatory mediators [9, 38-40] could have explained the observed systemic hemodynamic effects of haemofiltration. Finally, removal of sedative drugs leading to an increased sympathetic drive of animals cannot be completely excluded.

The apparent dissociation between the salutary effects of haemofiltration on vasopressor requirement and the totally absent improvement in other biological systems is a striking and key finding of this study. In this context, our experiment is the first and only study so far to assess the effects of haemofiltration on sepsis-mediated disturbances of microvascular perfusion and energy metabolism in different vital organs. Using laser Doppler flowmetry we 
did not observe any positive impact of haemofiltration on ileal mucosal and renal cortex microvascular perfusion. Since direct visualization of the ileal mucosal microcirculation gave similar results, it is unlikely that we overlooked major microvascular effects. These findings might be of particular importance given the fact that early microcirculatory alterations are related to a poor prognosis in human sepsis [41]. Furthermore, taking into account a hypothesis that haemofiltration may have reduced the burden of various mediators which, in fact, affect cellular metabolism, a concordant amelioration in markers reflecting metabolic stress would have been expected. Nonetheless, the progressive changes in hepato-splanchnic lactate/pyruvate and ketone bodies ratios indicating a deranged cytosolic and mitochondrial redox potential, respectively [42], clearly argue against an improvement in cellular energy balance with haemofiltration. Our results are in sharp contrast to data from Li et al. [43] who demonstrated that HVHF (100 ml/kg per hour) improved myocardial mitochondrial complex I activity in porcine peritonitis. These apparent dissociations are most likely due to the substantial differences in the model and study design used. While animals in the above mentioned study exhibited severe hypodynamic hypotensive shock and were studied within $12 \mathrm{~h}$ since the induction of peritonitis, we evaluated the effects of haemofiltration over $22 \mathrm{~h}$ of progressive hyperdynamic sepsis. Moreover, the prophylactic timing of intervention ( $1 \mathrm{~h}$ after the insult) and avoidance of vasopressors in the study by $\mathrm{Li}$ et al. make both studies difficult to compare. Given the limitations of our methodology, we were unable to determine whether or not the failure of haemofiltration to counteract the sepsisinduced microvascular and metabolic derangements is attributable to the limited capacity of plasma water exchange to affect the tissue or even cellular concentrations of sepsis mediators [44-46].

The endothelial cells represent a crucial target organ for the action of inflammatory mediators and are believed to be one of the key elements in the pathophysiology of sepsis [47, 48]. To the best of our knowledge, no data is available to document the capacity of haemofiltration to modulate sepsis-induced endothelial and coagulation dysfunction. In our study haemofiltration did not ameliorate the peritonitis-induced increase in plasma $\mathrm{vWf}$, a generally accepted marker of endothelial injury [49]. Likewise, the progressive fall in platelet count, probably as a result of disseminated intravascular coagulation, was not influenced by haemofiltration. The contribution of the extracorporeal circuit to these changes seems unlikely as haemofiltration did not influence the studied variables in sham-operated animals. These results are corroborated by our clinical study demonstrating that continuous hemodiafiltration neither modified plasma vWf levels nor affected platelet count [50].

While all previous experimental studies compared the effects of HVHF either with untreated subjects or with low-volume haemofiltration $(20 \mathrm{ml} / \mathrm{kg}$ per hour), our study is the first to seeking whether increasing the dose of plasma water exchange confers an additional benefit over the currently accepted standard haemofiltration dose, i.e. $35 \mathrm{ml} / \mathrm{kg}$ per hour. Although our observation corroborates recent reports, both from animal [16, 19, 20, 27] and clinical [9-14] trials, demonstrating a favorable effect on systemic haemodynamic, an ultrafiltration rate of $100 \mathrm{ml} /$ $\mathrm{kg}$ per hour failed to be superior over $35 \mathrm{ml} / \mathrm{kg}$ per hour in its capability to ameliorate disturbances in other mutually independent biological systems. These results could indicate, among other things, that the value of haemodynamic improvement as a surrogate marker for efficacy of haemofiltration might be ambiguous. Albeit indirectly, our findings also argue in support of the notion that haemofiltration appears to be the most effective when applied as pre-treatment or concomitantly with the septic or endotoxemic insult $[21,51,52]$, which might limit the clinical applicability.

Although our model replicates many of the features of human sepsis, there are some limitations that should be discussed. Antibiotic therapy that would bring the model closer to clinical standard was not used in this model. However, our model was designed to induce hyperdynamic sepsis with increasing severity over time. The antibiotic therapy was expected to blunt the host response to peritonitis, thereby attenuating the development of full manifestation of a true clinical septic response over the 22-h period as used in our experiment. Moreover, the study was not designed to evaluate survival rate but, rather, to focus on different and independent putative biological targets of haemofiltration. Furthermore, we can only speculate whether the observation study time was sufficient for detecting significant changes in the physiological end-points measured. Finally, since the severity of septic shock could be classified as moderate in our model, we cannot exclude different magnitude of the treatment response in more profound shock state.

In summary, in this model of septic shock secondary to peritonitis early HVHF proved superior to standard modality in preventing the development of septic hypotension. Nevertheless, the favorable effects of haemofiltration on vasopressor requirement did not translate into improved microvascular, metabolic and endothelial function, at least within the timeframe of the study and severity of the model.

Acknowledgments We thank L. Trefil, L. Vit and L. Reifova for their skillful technical assistance. Supported by research project MSM 0021620819 (Replacement of and support to some vital organs).

Open Access This article is distributed under the terms of the Creative Commons Attribution Noncommercial License which permits any noncommercial use, distribution, and reproduction in any medium, provided the original author(s) and source are credited. 


\section{References}

1. Cariou A, Vinsonneau C, Dhainaut JF (2004) Adjunctive therapies in sepsis: an evidence-based review. Crit Care Med 32:S562-S570

2. Honoré PM, Joannes-Boyau O, Gressens B (2007) Blood and plasma treatments: the rationale of high-volume haemofiltration. Contrib Nephrol 156:387-395

3. Bellomo R, Honoré PM, Matson J, Ronco C, Winchester J (2005) Extracorporeal blood treatment (EBT) methods in SIRS/Sepsis. Int J Artif Organs 28:450-458

4. Cole L, Bellomo R, Hart G, Journois D, Davenport P, Tipping P, Ronco C (2002) A phase II randomized, controlled trial of continuous haemofiltration in sepsis. Crit Care Med 30:100-106

5. Ronco C, Bellomo R, Homel P, Brendolan A, Dan M, Piccinni P, La Greca G (2000) Effects of different doses in continuous veno-venous haemofiltration on outcomes of acute renal failure: a prospective randomised trial. Lancet 356:26-30

6. Saudan P, Niederberger M, De Seigneux S, Romand J, Pugin J, Perneger T, Martin PY (2006) Adding a dialysis dose to continuous haemofiltration increases survival in patients with acute renal failure. Kidney Int 70:1312-1317

7. Schiffl H, Lang SM, Fischer R (2002) Daily hemodialysis and the outcome of acute renal failure. N Engl J Med 31:305-310

8. Palevsky PM, Zhang JH, O'Connor TZ, Chertow GM, Crowley ST, Choudhury D, Finkel K, Kellum JA, Paganini E, Schein RM, Smith MW, Swanson KM, Thompson BT, Vijayan A, Watnick S, Star RA, Peduzzi P (2008) The VA/NIH acute renal failure trial network intensity of renal support in critically ill patients with acute kidney injury. N Engl J Med 359:7-20

9. Cole L, Bellomo R, Journois D, Davenport P, Baldwin I, Tipping P (2001) High-volume haemofiltration in human septic shock. Intensive Care Med 27:978-986

10. Honore PM, Jamez J, Wauthier M, Lee PA, Dugernier T, Pirenne B, Hanique G, Matson JR (2000) Prospective evaluation of short-term, high-volume isovolemic haemofiltration on the hemodynamic course and outcome in patients with intractable circulatory failure resulting from septic shock. Crit Care Med 28:3581-3587
11. Ratanarat R, Brendolan A, Piccinni P, Dan M, Salvatori G, Ricci Z, Ronco C (2005) Pulse high-volume haemofiltration for treatment of severe sepsis: effects on haemodynamics and survival. Crit Care 9:294-302

12. Oudemans-van Straaten HM, Bosman RJ, van der Spoel JI, Zandstra DF (1999) Outcome of critically ill patients treated with intermittent high-volume haemofiltration: a prospective cohort analysis. Intensive Care Med 25:814821

13. Joannes-Boyau O, Rapaport S, Bazin R, Fleureau C, Janvier G (2004) Impact of high volume haemofiltration on hemodynamic disturbance and outcome during septic shock. ASAIO J 50:102109

14. Piccinni P, Dan M, Barbacini S, Carraro R, Lieta E, Marafon S, Zamperetti N, Brendolan A, D'Intini V, Tetta C, Bellomo R, Ronco C (2006) Early isovolaemic haemofiltration in oliguric patients with septic shock. Intensive Care Med 32:80-86

15. Cornejo R, Downey P, Castro R, Romero C, Regueira T, Vega J, Castillo L, Andresen M, Dougnac A, Bugedo G, Hernandez G (2006) High-volume haemofiltration as salvage therapy in severe hyperdynamic septic shock. Intensive Care Med 32:713-722

16. Grootendorst AF, van Bommel EF, van der Hoven B, van Leengoed LA, van Osta AL (1992) High volume haemofiltration improves right ventricular function in endotoxininduced shock in the pig. Intensive Care Med 18:235-240

17. Ullrich R, Roeder G, Lorber C, Quezado ZM, Kneifel W, Gasser H, Schlag G, Redl H, Germann P (2001) Continuous venovenous haemofiltration improves arterial oxygenation in endotoxin-induced lung injury in pigs. Anesthesiology 95:428-436

18. Yekebas EF, Eisenberger CF, Ohnesorge H, Saalmüller A, Elsner HA, Engelhardt M, Gillesen A, Meins J, The M, Strate T, Busch C, Knoefel WT, Bloechle C, Izbicki JR (2001) Attenuation of sepsis-related immunoparalysis by continuous venovenous haemofiltration in experimental porcine pancreatitis. Crit Care Med 29:1423-1430

19. Bellomo R, Kellum JA, Gandhi CR, Pinsky MR, Ondulik B (2000) The effect of intensive plasma water exchange by haemofiltration on haemodynamics and soluble mediators in canine endotoxemia. Am J Respir Crit Care Med 161:1429-1436
20. Rogiers P, Zhang H, Smail N, Pauwels D, Vincent JL (1999) Continuous venovenous haemofiltration improves cardiac performance by mechanisms other than tumor necrosis factor-alpha attenuation during endotoxic shock. Crit Care Med 27:1848-1855

21. Bouman CS, Oudemans-van Straaten HM, Schultz MJ, Vroom MB (2007) Haemofiltration in sepsis and systemic inflammatory response syndrome: the role of dosing and timing. J Crit Care 22:1-12

22. Matejovic M, Krouzecky A, Martinkova V, Rokyta R Jr, Kralova H, Treska V, Radermacher P, Novak I (2004) Selective inducible nitric oxide synthase inhibition during long-term hyperdynamic porcine bacteremia. Shock 21:458-465

23. Matejovic M, Krouzecky A, Martinkova V, Rokyta R Jr, Radej J, Kralova H, Treska V, Radermacher P, Novak I (2005) Effects of tempol, a free radical scavenger, on long-term hyperdynamic porcine bacteremia. Crit Care Med 33:1057-1063

24. Matejovic M, Krouzecky A, Rokyta R Jr, Radej J, Kralova H, Treska V, Radermacher P, Novak I (2007) Effects of combining inducible nitric oxide synthase inhibitor and radical scavenger during porcine bacteremia. Shock 27:61-68

25. Tugtekin IF, Radermacher $\mathrm{P}$, Theisen M, Matejovic M, Stehr A, Ploner F, Matura K, Ince C, Georgieff $\mathrm{M}$, Träger $\mathrm{K}$ (2001) Increased ileal-mucosalarterial $\mathrm{PCO}_{2}$ gap is associated with impaired villus microcirculation in endotoxic pigs. Intensive Care Med 27:757-766

26. De Backer D, Hollenberg S, Boerma C, Goedhart P, Büchele G, Ospina-Tascon G, Dobbe I, Ince C (2007) How to evaluate the microcirculation: report of a round table conference. Crit Care 10;11:R101

27. Yekebas EF, Strate T, Zolmajd S, Eisenberger CF, Erbersdobler A, Saalmüller A, Steffani K, Busch C, Elsner HA, Engelhardt M, Gillesen A, Meins J, The M, Knoefel WT, Izbicki JR (2002) Impact of different modalities of continuous venovenous haemofiltration on sepsis-induced alterations in experimental pancreatitis. Kidney Int 62:1806-1818

28. Asfar P, Hauser B, Radermacher P, Matejovic M (2006) Catecholamines and vasopressin during critical illness. Crit Care Clin 22:131-149

29. Landry DW, Oliver JA (2001) The pathogenesis of vasodilatory shock. N Engl J Med 345:588-595 
30. Takakura K, Taniguchi T, Muramatsu I, Takeuchi K, Fukuda S (2002) Modification of alfa-1 adrenoreceptors by peroxynitrite as a possible mechanism of systemic hypotension in sepsis. Crit Care Med 30:894-899

31. Takakura K, Xiaohong W, Takeuchi K, Yasuda Y, Fukuda S (2003)

Deactivation of noradrenaline by peroxynitrite as a new pathogenesis in the hypotension of septic shock. Anestesiology 98:928-934

32. Macarthur H, Couri DM, Wilken GH, Westfall TC, Lechner AJ, Matuschak GM, Chen Z, Salvemini D (2003) Modulation of serum cytokine levels by a novel superoxide dismutase mimetic, M40401, in an Escherichia coli model of septic shock: correlation with preserved circulating catecholamines. Crit Care Med 31:237-245

33. Rokyta R Jr, Matejovic M, Krouzecky A, Opatrny K Jr, Ruzicka J, Novak I (2004) Effects of continuous venovenous haemofiltration-induced cooling on global haemodynamics, splanchnic oxygen and energy balance in critically ill patients. Nephrol Dial Transplant 19:623-630

34. Marsh JD, Margolis TI, Kim D (1988) Mechanism of diminished contractile response to catecholamines during acidosis. Am J Physiol 254:H20-H27

35. Kellum JA, Song M, Venkataraman R (2004) Effects of hyperchloremic acidosis on arterial pressure and circulating inflammatory molecules in experimental sepsis. Chest 125:243248

36. Rogiers P, Sun Q, Dimopoulos G, Tu Z, Pauwels D, Manhaeghe C, Su F, Vincent JL (2006) Blood warming during haemofiltration can improve haemodynamics and outcome in ovine septic shock. Anesthesiology 104:1216-1222
37. Ronco C, Tetta C, Mariano F, Wratten ML, Bonello M, Bordoni V, Cardona X, Inguaggiato P, Pilotto L, d'Intini V, Bellomo R (2003) Interpreting the mechanisms of continuous renal replacement therapy in sepsis: the peak concentration hypothesis. Artif Organs 27:792-801

38. Hoffmann JN, Hartl WH, Deppisch R, Faist E, Jochum M, Inthorn D (1996) Effect of haemofiltration on haemodynamics and systemic concentrations of anaphylatoxins and cytokines in human sepsis. Intensive Care Med 22:1360-1367

39. Silvester W (1997) Mediator removal with CRRT: complement and cytokines. Am J Kidney Dis 30:S38S43

40. Wang Y, Liu Y, Sarker KP, Nakashima M, Serizawa T, Kishida A, Akashi M, Nakata M, Kitajima I, Maruyama I (2000) Polymyxin B binds to anandamide and inhibits its cytotoxic effect. FEBS Lett 24(470):151-155

41. Trzeciak S, Dellinger RP, Parrillo JE, Guglielmi M, Bajaj J, Abate NL, Arnold RC, Colilla S, Zanotti S, Hollenberg SM (2007) Microcirculatory alterations in resuscitation and shock investigators early microcirculatory perfusion derangements in patients with severe sepsis and septic shock: relationship to haemodynamics, oxygen transport, and survival. Ann Emerg Med 49:88-98

42. Leverve XM (1999) From tissue perfusion to metabolic marker: assessing organ competition and cooperation in critically ill patients? Intensive Care Med 25:890-892

43. Li CM, Chen JH, Zhang P, He Q, Yuan J, Chen RJ, Cheng XJ, Tan HZ, Yang Y (2007) Continuous veno-venous haemofiltration attenuates myocardial mitochondrial respiratory chain complexes activity in porcine septic shock. Anaesth Intensive Care 35:911919
44. Joannes-Boyau O, Honore PM, Boer W (2006) Haemofiltration: the case for removal of sepsis mediators from where they do harm. Crit Care Med 34:22442246

45. Honoré PM, Matson JR (2004) Extracorporeal removal for sepsis: acting at the tissue level-the beginning of a new era for this treatment modality in septic shock. Crit Care Med 32:896897

46. Ronco C (2007) The immunomodulatory effect of extracorporeal therapies in sepsis: a reconciliation of three theories. Int $\mathbf{J}$ Artif Organs 30:855-857

47. Hack CE, Zeerleder S (2001) The endothelium in sepsis: source of and target for inflammation. Crit Care Med 29:S21-S27

48. Schouten M, Wiersinga WJ, Levi M, van der Poll T (2008) Inflammation, endothelium, and coagulation in sepsis. J Leukoc Biol 83:536-545

49. Reinhart K, Bayer O, Brunkhorst F, Meisner M (2002) Markers of endothelial damage in organ dysfunction and sepsis. Crit Care Med 30:S302-S312

50. Polanska K, Opatrny K Jr, Rokyta R Jr, Krouzecky A, Vit L, Kasal E (2006) Effect of regional citrate anticoagulation on thrombogenicity and biocompatibility during CVVHDF. Ren Fail 28:107-118

51. Honore' PM, Joannes-Boyau O, Merson L, Boer W, Piette V, Galloy AC, Janvier G (2006) The big bang of haemofiltration: the beginning of a new era in the third millennium for extracorporeal blood purification! Int J Artif Organs 29:649-659

52. Mink SN, Li X, Bose D, Gu M, Liu G, Jacobs H, Light RB (1999) Early but not delayed continuous arteriovenous haemofiltration improves cardiovascular function in sepsis in dogs. Intensive Care Med 25:733-743 